ESJ Humanities

\title{
Analyse de l'étendue des privilèges et immunités diplomatiques
}

\author{
Docteur Jean de Dieu Ndikumana \\ Enseignant-chercheur à l'École Nationale d'administration \\ Professeur visiteur à l'Université lumière de Bujumbura et à Bujumbura \\ International University
}

Doi: $10.19044 /$ esj.2021.v17n2p79

Submitted: 16 December 2020

Accepted: 21 January 2021

Published: 31 January 2021
Copyright 2021 Author(s)

Under Creative Commons BY-NC-ND

4.0 OPEN ACCESS

Cite As:

Ndikumana J.D. (2021). Analyse de l'étendue des privilèges et immunités diplomatiques. European Scientific Journal, ESJ, 17(2), 79. https://doi.org/10.19044/esj.2021.v17n2p79

\section{Resume}

La restriction de liberté de voyager d'un pays à l'autre pour les hautes autorités des Etats à cause de la pandémie de Covid-19 a diminué les échanges relationnels entre les Etats d'une part, et d'autre part a beaucoup ravivé les activités des diplomates qui deviennent intense et incontournable pendant ces moments où les pays se ferment sur eux-mêmes. Les pays du monde entier mènent des relations internationales au moyen de la diplomatie. Ils envoient des agents dans des missions diplomatiques pour bien accomplir leur tâche. Pour mener bien leur mission, ils ont besoin d'un environnement favorable que leur offre le pays d'accréditation. Cette réalité se définit en termes de privilèges et immunités diplomatiques. La question de la pratique s'impose dans cette réflexion de manière suivante : la pratique de ces privilèges et immunités est-elle absolue? C'est dans ce contexte que s'inscrit cette étude qui vise à analyser les fondements des privilèges et immunités octroyés au corps diplomatique par la convention de Vienne de 1961 et leur mis en application au quotidien. Cette réflexion a démontré qu'il existe des limites liées à la pratique des privilèges et immunités. Pour y parvenir, une méthode documentaire est privilégiée. La recherche documentaire choisie pour cette étude a permis d'exploiter les livres, les articles, les rapports ainsi que divers documents disponibles sur Internet pour y tirer des informations nécessaires relatives à ce sujet. Cette étude a terminé sa réflexion par une conclusion qui 
dégage des approches de solutions pour les limites identifiées.

Mots clés : Diplomatie, Privilèges et Immunités, Convention de Vienne de 1961

\title{
Analysis of the Scope of Diplomatic Privileges and Immunities
}

\author{
Docteur Jean de Dieu Ndikumana \\ Enseignant-chercheur à l'École Nationale d'administration \\ Professeur visiteur à l'Université lumière de Bujumbura et à Bujumbura \\ International University
}

\begin{abstract}
The restriction of the movement of the authorities of different countries due to the Covid-19 pandemic has both reduced the relationships between these States and has revived the activities of diplomats which become intense and unavoidable during these times since countries are being confined. While many Countries conduct international diplomatic relations, they send agents to diplomatic missions to achieve their targets. In order to carry out their mission successfully, they need a favorable environment offered by their receiving countries. This reality is defined in terms of diplomatic privileges and immunities. The question of practice arises in this reflection as follows: Is the practice of these privileges and immunities totally absolute? Therefore, it is in this context that this study takes place and it is meant to analyze the foundations of the privileges and immunities which are granted to the diplomatic corps in accordance with the 1961 Vienna Convention. This reflection has shown that there are limits linked to the practice of privileges and immunities. To achieve this, a documentary method is preferred. The documentary research chosen for this study made it possible to use the books, articles, reports and various documents available on the Internet to extract the necessary information on this subject. This study ended its reflection with a conclusion which proposed the approaches of possible solutions towards the identified limitations.
\end{abstract}

Keywords: Diplomacy, Privileges and immunities, Vienna convention 1961

\section{Introduction}

Le monde traverse des moments difficiles où la pandémie du Covid19 a réduit sensiblement la liberté de voyager surtout d'un pays à l'autre. Jusqu'alors, les relations interétatiques étaient menées d'une part par les hautes autorités des pays et d'autre part par leurs représentants se trouvant 
dans des pays accréditaires. Les exigences de lutte contre la propagation du Covid-19 ont introduit de nouvelles façons de faire. Actuellement, par contrainte, beaucoup de réunions s'organisent par le biais de vidéoconférences tant au niveau bilatéral qu'au multilatéral, régional et international. Le virtuel a pris le devant sur le travail en présentiel. Néanmoins, le métier de diplomatie a toujours témoigné et témoigne encore l'importance de la présence physique comme exigence du métier. À titre d'exemple, les négociations se feraient mieux en présentiel que par vidéoconférence. C'est pour cette raison que l'activité diplomatique recouvre toute son importance en cette période de pandémie. Les diplomates étant sur place dans les pays accréditaires, ils sont appelés, par les exigences du contexte pandémique, à jouer un rôle plus prépondérant dans leur art de représentativité. Cela permet de combler les absences éventuelles des déplacements de leurs chefs hiérarchiques et démontre l'actualité et l'intérêt de la présente discussion qui porte sur l'analyse de l'étendue des privilèges et immunités des agents diplomatiques.

Les relations diplomatiques et consulaires entre les peuples existent depuis longtemps. Le droit international les a adoptées, organisées, mais non instituées. Après apparition du phénomène étatique, la pratique révèle que le critère le plus sûr de la souveraineté d'un État est le fait qu'il entre effectivement, par l'intermédiaire de ses propres agents et sur un pied d'égalité, dans les relations diplomatiques et consulaires avec d'autres États souverains et qu'il soit représenté auprès des organisations internationales et participe à leurs activités (Nguyen, Daillier et al., 1999). Les agents diplomatiques assurent les relations officielles entre les États. Ils représentent leurs pays et exécutent la politique étrangère de leurs pays d'origine. Ce sont les organes officiels du système moderne des relations internationales. Il est admis également aujourd'hui un droit de légation au profit des Organisations Internationales à l'instar des États. Dans son exercice, le droit de légation comporte deux aspects : d'un côté le droit de légation active qui est celui d'envoyer des représentants auprès d'États tiers ou d'organisations internationales et de l'autre côté, le droit de légation passive consistant à recevoir les représentants diplomatiques des pays étrangers. D'après cette conception, le droit de légation serait un droit naturel alors que les privilèges et immunités découleraient de celui-ci. Gentili affirmait notamment que le droit d'ambassade était «en vertu de la Divine Providence, immuable, d'application universelle, et admis et reconnu même par les peuples barbares » (Gentili, 1954).

Cette théorie n'est guère satisfaisante dans la mesure où le droit de légation diplomatique est un droit positif et il n'apparaît point nécessaire de recourir à un droit naturel que les internationalistes contemporains rejettent (Philippe, 1954). 
La mission des représentants des États est d'informer leur propre gouvernement et celui auprès duquel ils sont accrédités sur la vie politique, économique et sociale de l'État accréditaire ainsi que leur pays d'origine. Ils tâchent d'éliminer les obstacles et les incompréhensions, de développer la coopération entre les peuples au niveau culturel, scientifique, commercial, de préparer et parfois de signer des conventions internationales (Papini et Cortese, 1972). Les agents et la mission diplomatique se trouvent dans une situation très particulière. Ils constituent des moyens pour l'État accréditant d'exercer une mission de service public sur le territoire de l'État accréditaire. Cette position spéciale conduit à reconnaître aux uns et aux autres des garanties exceptionnelles permettant au moins ou facilitant l'accomplissement de cette mission. On désigne ces facilités par l'expression «privilèges et immunités » (Nguyen, Daillier et al. 1999).

Pour faciliter et rendre efficace leur travail, la Convention de Vienne sur les relations diplomatique et consulaire a étendu les privilèges et immunités aux membres de leur famille. Cela dans le but de tranquilliser les diplomates pendant leur séjour sachant que ces membres de famille ne peuvent être objet de fouilles ou d'arrêts par la police ou la justice du pays hôte (Convention de Vienne, 1961).

Tous les États qui accueillent des représentations étrangères et des organisations internationales leur accordent des privilèges, immunités et facilités. Ceux-ci sont étendus aux membres de leur personnel. Ce statut découle des dispositions de la Convention de Vienne de 1961 et de 1963 et des accords subséquents en matière de privilèges, immunités et facilités. Les privilèges et immunités accordés aux membres du personnel ne sont toutefois pas établis en vue de conférer à ceux qui en bénéficient des avantages personnels. Ils sont institués uniquement afin d'assurer, en toute circonstance, le fonctionnement de la mission ou de l'organisation et l'indépendance des personnes concernées dans l'exercice de leurs fonctions. Sans préjudice de leurs privilèges et immunités, les bénéficiaires de ce statut ont le devoir de respecter les lois et règlements de l'État hôte.

Au-delà des agents diplomatiques, les privilèges et immunités s'étendent à une autre catégorie de hautes autorités des États en déplacement en dehors de leur territoire. En l'occurrence, il s'agit des chefs d'État, des chefs de gouvernements, ainsi que les ministres des Affaires étrangères. Cette catégorie des bénéficiaires des immunités n'étant pas couverte par la Convention de Vienne, ne concerne pas l'objet de cette étude.

Ainsi, l'octroi des privilèges et immunités aux agents diplomatiques comme prévu dans la Convention de Vienne sur les relations diplomatiques de 1961 vise à garantir que les missions diplomatiques et de représentation des États puissent exercer leur fonction avec efficacité. Les privilèges et immunités visent à assurer la prédominance de l'intérêt de la fonction des 
diplomates. Lesdits privilèges et immunités n'ont pas pour objet d'avantager leurs titulaires, mais d'assurer l'accomplissement efficace des fonctions des missions diplomatiques en tant que représentant des États (Convention de Vienne 1961).

À cet effet, l'immunité diplomatique porte sur un ensemble de privilèges accordés par le pays hôte aux membres du corps diplomatique d'un pays tiers et à leur famille durant leur séjour en poste. Cependant, à côté de ce qui est prévu par la Convention de Vienne, la jurisprudence consécutive à certains cas d'incidents diplomatiques a montré que la mise en pratique des privilèges et immunités diplomatiques est très délicate. En témoigne le cas du journaliste Saoudien Jamal Khashoggi qui s'est réfugié le 2 octobre 2018 dans le consulat d'Arabie Saoudite accrédité en Turquie et il est retrouvé mort le même jour. Cela témoigne que malgré l'existence des conventions internationales sur l'inviolabilité des locaux, la pratique sur terrain prouve le contraire dans le cas d'espèce. Pourtant la Convention de Vienne de 1961 sur les relations diplomatiques précise en son article 22.1 qu'une intervention des forces de l'ordre du pays hôte est possible «avec le consentement du chef de la mission », ce qui n'a pas été le cas dans l'affaire de Jamal Khashoggi.

En dépit des incidents et des difficultés qui caractérisent, la mise en œuvre de ces privilèges et immunité, le constat est aussi que ces derniers ne sont pas absolus. Les pratiques des États démontrent des manquements au niveau de l'effectivité de l'application des textes. D'où cette étude a pour objectif d'analyser en profondeur ces privilèges et immunités en vue d'en dégager des limites à partir desquelles des solutions seront proposées.

Les analyses permettront de répondre à la question de savoir l'étendue des privilèges et immunités garantis aux diplomates en mission. Pour mener cette réflexion centrée sur l'analyse de l'étendue des privilèges et immunités diplomatiques, cinq points sont abordés. L'étude traite d'abord l'introduction (I). Le point suivant exploite les fondements des privilèges et immunités (II) en mettant l'accent sur l'incarnation du souverain à l'étatisation de la mission diplomatique (II.1), prééminence de l'intérêt de la fonction sur le prestige personnel du diplomate (II.2), de la présence physique à l'absence juridique du diplomate dans l'État accréditaire (II.3). Ensuite suit le point sur la relativité des privilèges et immunités diplomatiques (III) que les développements déclinent en six points suivants : prépondérance de la sécurité et de l'ordre public sur l'inviolabilité (III.1), étendue des privilèges et immunités en cas de possession de résidence ou de nationalité du pays accréditaire (III.2), la temporalité des privilèges et immunités diplomatiques (III.3), circonscription spatiale des privilèges et immunités diplomatiques (III.4), les contrôles sur la personne et des fouilles de bagages de diplomates dans les aéroports (III.5) et protection incidente des correspondances et valises diplomatiques (III.6). Enfin, l'étude analyse le particularisme de la protection 
des locaux des missions diplomatiques et de l'imposition (IV) avant de clore par une conclusion $(\mathrm{V})$.

\section{Fondement des privilèges et immunités diplomatiques}

Les privilèges et immunités diplomatiques incarnent l'existence d'un souverain conduisant à l'étatisation de la mission diplomatique. La question de fondement des privilèges et immunités a pu susciter quelques grandes théories qu'il semble très indispensable d'analyser. Ce sont des instruments qui consacrent la prééminence des intérêts de l'État sur le privilège personnel du diplomate. Celui-ci est certes présent physiquement sur le territoire de l'État accréditaire mais juridiquement, il en dépend moins pour certains aspects.

\section{1 De l'incarnation du souverain à l'étatisation de la mission diplomatique}

Le diplomate incarne le souverain et ceci remonte du moyen âge où les rapports internationaux étaient considérés comme des rapports personnels entre princes et rois. Les délégués de ces rois étaient considérés comme leurs représentants personnels et toute violence ou offense à leur dignité était estimée comme offense au souverain lui-même. Bien plus, étant donné l'idée de l'égalité des États et des princes, qui à l'époque personnifiait l'État, il en résultait qu'une loi édictée par un souverain ne pouvait s'appliquer à un autre souverain et conséquemment à son représentant direct (Burlama qui, 1766). La convention de Vienne de 1961 sur les relations diplomatiques a matérialisé cette pratique de jadis en octroyant les privilèges et immunités aux agents diplomatiques dans l'État accréditaire.

Cette idée soutenue par Burlama fut particulièrement bien exprimée par Montesquieu. Celui-ci déclare : « Le droit des gens a voulu que les princes s'envoyassent des ambassadeurs, et la raison, tirée de la nature de la chose, n'a pas permis que ces ambassadeurs dépendissent du souverain chez qui ils sont envoyés ni de ses tribunaux. Ils sont la parole du prince qui les envoie et cette parole doit être libre. Que s'ils abusent de leur côté représentatif on le fait cesser en les envoyant chez eux. » (Montesquieu, 1748 cités par Philipe, 1964). C'est de cette idée que toute offense faite à l'ambassadeur est considérée comme une atteinte à la dignité personnelle du souverain dont il est l'envoyé. Cette conception a inspiré le statut de 1708 de la reine Anne sur les immunités diplomatiques qui est peut-être une des premières lois internes promulguées en la matière (Bély, 2012).

Une telle théorie devait être abandonnée par la suite, car elle ne correspondait plus à la réalité. En premier lieu, à cause du changement des caractéristiques des souverains, les États ne sont plus considérés comme la propriété des princes, mais de la population tout entière. Cette nouvelle 
conception a fait évoluer le caractère représentatif des diplomates en le détachant de la personne du roi ou du prince. De plus, si cette théorie était correcte, les chefs d'État devraient jouir des mêmes immunités que leurs représentants, ce qui n'est pas toujours le cas. En outre, si c'est seulement en sa qualité personnelle de représentant d'un État que le diplomate jouit de ce statut privilégié, pour quelle raison sa famille, qui n'a aucun caractère représentatif, bénéficie-t-elle de ce même statut? Les immunités et privilèges diplomatiques doivent être considérés comme des instruments d'action de l'État dans sa quête des relations avec les autres États ou organisations internationales. Comme l'écrit Fauchille, «[L] es immunités diplomatiques ayant pour raison d'être le caractère représentatif des agents, c'est-à-dire la nécessité de leur assurer l'indépendance nécessaire à l'exercice de leurs fonctions ». (Fauchille, 1922). Cette étude en conclut que ce sont des moyens conférés aux diplomates dans l'intérêt de sa fonction étatique qu'il exerce à l'étranger (Philippe, 1964).

\section{2 Prééminence de l'intérêt de la fonction sur le prestige personnel des diplomates}

L'immunité n'est pas rattachée à un homme, mais à une fonction : on ne parle pas de l'immunité de $\mathrm{X}$ ou de $\mathrm{Y}$, mais de l'immunité du représentant d'un État. «C'est en leur qualité d'organes de l'État que différentes catégories d'agents sont au bénéfice d'immunités diverses, dont il a été maintes fois souligné qu'elles ne sont pas instituées pour avantager des individus, mais pour assurer l'accomplissement efficace des fonctions officielles de la mission ». (Convention de Vienne, art. 25). L'immunité fonctionnelle porte parfois aussi le nom d'immunité rationae materiae (immunité matérielle), que l'on oppose à l'immunité personnelle (rationae personae). Casseses et DelmasMarly distinguent ces deux immunités comme suit: «Les premières s'appliquent à toute personne agissant en sa qualité officielle de représentant d'un État: limitées aux actes liés aux fonctions (compétence rationae materiae), elles ont un caractère permanent et font obstacle aux poursuites même après que les fonctions eurent pris fin alors que les secondes sont plus larges en ce qu'elles peuvent couvrir des comportements à caractère privé, protègent la personne (compétence rationae personae), notamment lors de ses déplacements à l'étranger, mais elles sont temporaires et prennent fin avec la cessation des fonctions ». (Casseses et Delmas-Marly, 2002). Vattel explique le rôle de ces immunités en ces termes : «[...] les ambassadeurs et autres ministères publics sont des instruments nécessaires à l'entretien de cette Société Générale, de cette correspondance mutuelle des nations. Mais leur ministère ne peut atteindre la fin à laquelle il est destiné, s'il n'est muni de toutes les prérogatives capables d'en assurer le succès légitime, de le faire exercer en toute sécurité librement et fidèlement. Le même droit des gens qui 
oblige les nations à admettre les ministères étrangers, les oblige donc aussi manifestement à recevoir ces ministères avec tous les droits qui leur sont nécessaires, tous les privilèges qui assurent l'exercice de leurs fonctions ». (Vattel, 1830). De ce passage, on en déduit qu'un représentant diplomatique ne pourra exercer ses fonctions que s'il est pleinement indépendant de l'État qui le reçoit. Cette théorie est aujourd'hui largement admise dans la doctrine (Philippe, 1964). Ces fonctions renvoient à des tâches aussi diverses que celles d'entretenir des relations privilégiées avec les élites du pays, de choisir des personnes-ressources à inviter lors des réceptions et événements organisés par l'ambassade, ou encore d'honorer des invitations à des manifestations officielles du pays d'accréditation.

Loin du prestige personnel du fonctionnaire étatique, l'intérêt de l'accomplissement des fonctions de la mission et n'appartient pas seulement à l'État accréditant, mais aussi à l'État accréditaire qu'à toute la communauté des États. Pour ceux-ci, il est nécessaire que leurs relations diplomatiques soient assurées, mais il est bon aussi que les rapports diplomatiques s'effectuent pacifiquement (Philippe, 1964).

Cette idée est exprimée de manière particulièrement heureuse par Guggenheim quand il déclare que «[c]» est dans l'intérêt collectif des relations internationales que les représentants diplomatiques jouissent de certains privilèges et immunités ». (Guggenheim, 1953).

Cette théorie de l'intérêt de la fonction explique la raison pour laquelle la famille du diplomate jouit aussi d'un statut privilégié. En effet celui-ci ne pourrait accomplir librement sa mission si sa famille pouvait faire l'objet de mesures de contrainte.

Au regard de cette théorie de l'intérêt, les immunités appartiennent à l'État accréditant qui peut les lever s'il estime que leur disparition dans un cas donné n'est pas de nature à nuire à l'accomplissement de la mission de l'agent. (Philippe, 1964). Cette idée est sans doute exacte et se trouve probablement à la base de la disparition de certains privilèges tels que la franchise des quartiers, le droit d'asile, le droit pour le chef de mission de juger le personnel de l'ambassade, etc. D'autres privilèges pourraient encore à l'heure actuelle disparaître sans inconvénient majeur. Certes, ils peuvent subsister sur la base du statut privilégié des diplomates (Philippe, 1964).

Le domaine des relations internationales, du fait de son absence d'organisation et d'une autorité supérieure, a toujours été un domaine où la réciprocité a joué un rôle. Le comportement irrégulier d'un Etat entraîne le plus souvent des représailles semblables de la part de l'État victime de ces actes irréguliers. Cela est particulièrement vrai dans le domaine du droit diplomatique, car si un État accorde un traitement favorable aux diplomates d'un autre, c'est qu'il s'attend à ce que ses propres diplomates jouissent d'un même traitement. Inversement, tout traitement défavorable entrainera une 
réaction de la part de l'État dont les diplomates sont amenés à le subir. C'est une des raisons pour lesquelles les atteintes aux privilèges et immunités des diplomates sont assez rares. Cette théorie de la réciprocité est importante et semble constituer le pilier de la pratique diplomatique (Philippe, 1964). Les règles relatives aux privilèges sont des normes réciproques, c'est-à-dire que leur application est conditionnée par l'existence d'une règle semblable dans l'ordre juridique interne de l'autre État, mais cela est applicable uniquement pour les privilèges de courtoisie, c'est-à-dire ceux qui ne sont pas nécessaires à l'accomplissement des fonctions du diplomate.

L'application de cette règle de réciprocité conduit à des pratiques discriminatoires assez déplorables. Les diplomates accrédités dans un pays jouiront, selon l'État auquel ils appartiennent, de statuts privilégiés différents. Cette conséquence qui découle du principe de la réciprocité est assez grave. De représailles en représailles, on peut aboutir à rescinder les privilèges au minimum. Bien plus, certains États pourraient acquérir une tendance à décider unilatéralement quels sont les privilèges qu'ils accordent aux diplomates étrangers et quels sont ceux qu'ils leur refusent. Une telle attitude, et il faut y insister avec force, est tout à fait contraire au droit diplomatique. Dans ce cas, les États sont obligés, par l'ordre juridique international, d'accorder ces privilèges et immunités indépendamment de la manière dont un autre État les accorde. Si ce dernier agit de manière illicite, l'État pourra recourir aux sanctions prévues par le droit international et notamment à la rupture des relations diplomatiques (Philippe, 1964).

\section{3 De la présence physique à l'absence juridique du diplomate dans l'État accréditaire}

Selon la théorie dite d'exterritorialité, le diplomate échappe au pouvoir de commandement du souverain territorial par une fiction, en vertu de laquelle l'agent est censé n'avoir jamais quitté son territoire national. En d'autres termes, il exerce ses fonctions de diplomate accrédité auprès d'un État en restant dans son propre pays. Physiquement présent sur le territoire de l'État accréditaire, il en est juridiquement absent. De cette théorie de l'exterritorialité fictive, on est passé à la théorie de l'exterritorialité réelle en disant que l'ambassade devait être considérée comme une portion du territoire national (Philippe, 1964). Grocius exprime bien cette théorie en opinant que « [...] comme un ambassadeur représente, par une espèce de fiction, la personne même de son Maître, il est aussi regardé, par une fiction semblable, comme étant hors des terres de la puissance auprès de qui il exerce ses fonctions et de là vient qu'il n'est point tenu d'observer les lois civiles du pays étranger où il demeure en ambassade ». (Grotius, 1724).

La faiblesse de cette conception de Grocius est qu'elle peut faire croire que l'ambassadeur est au-dessus des lois du pays, ce qui est faux et contraire 
à la pratique internationale en la matière. L'emploi de ce terme d'exterritorialité est fort regrettable, car il risque de créer des confusions et faire croire à certains diplomates qu'ils sont au-dessus des lois ou, au profane, qu'un territoire extraterritorial est un territoire qui appartient à un autre État que celui où il se trouve. Ainsi, il serait souhaitable de la voir disparaître une fois pour toutes du langage diplomatique et juridique. À ce point de vue, la conférence de la convention de Vienne de 1961 a fait œuvre utile en supprimant dans la rédaction de la Convention tout emploi de ce terme (Philippe, 1964).

Hurst (1926) avait développé une théorie semblable à celle de Philippe en partant de l'idée que les agents diplomatiques étrangers sont reçus par l'Etat à la condition qu'ils ne seront pas soumis à sa juridiction. Bien plus, ils ne doivent aucune allégeance au souverain du pays où ils sont accrédités. L'auteur ajoute qu' «ils seront hors de sa juridiction, ils sont à l'abri de ses lois ». Mais là aussi, cette affirmation manque de logique et ne correspond pas à la réalité. Elle manque de logique, car si le diplomate n'est pas soumis aux lois, il n'a pas besoin d'immunités, et il est évident qu'il ne saurait être poursuivi devant les tribunaux pour violation d'une loi qui ne s'appliquerait pas à lui. La notion d'immunité devient dans ce cas inutile. Elle est fausse, car comme il a été déjà dit et répété, le diplomate est soumis à la législation locale, l'immunité n'a pour effet que de paralyser la sanction. Si l'immunité est levée, la loi reprendra toute sa vigueur et le diplomate peut être poursuivi devant les tribunaux locaux, ce qui ne serait pas possible si le diplomate n'était pas soumis à la loi au moment où il accomplissait l'acte illicite. Comme la doctrine de l'exterritorialité, cette théorie est dangereuse, car elle donne l'impression aux agents diplomatiques de n'être soumis à aucune loi (Philippe, 1964).

La doctrine de l'accord tacite fut soutenue par le duc d'Aiguillon considère que « [1] » immunité des ambassadeurs et autres ministres est fondée sur deux principes à savoir la dignité du caractère représentatif auquel ils participent plus au moins et sur la convention tacite qui résulte de ce qu'en admettant un ministre étranger, on reconnait les droits de l'usage que le droit des gens, lui accorde ». (Duc d'Aiguillon, ministre des Affaires étrangères de Louis XV en 1772).

Il en résulte que cette convention, étant bilatérale, disparaît dès que le diplomate abuse de ces privilèges, car en agissant ainsi il va évidemment à l'encontre des intentions des deux souverains. Une telle théorie n'est évidemment pas soutenable et ne s'explique que parce que le duc d'Aiguillon cherchait à justifier une violation des privilèges et immunités diplomatiques. $\mathrm{Si}$, à la base des relations diplomatiques, on trouve un accord qui n'est d'ailleurs pas toujours tacite, cet accord porte sur la création de ces relations diplomatiques, voire sur la personne de l'agent à envoyer, mais absolument pas sur le statut privilégié à lui accorder. Celui-ci est imposé aux États par des 
règles de droit. Si on acceptait la version du duc d'Aiguillon, l'agent diplomatique serait à la merci du gouvernement de l'État accréditaire qui pourrait facilement, sous un prétexte quelconque, déclarer la convention violée et donc poursuivre le diplomate par mesure de représailles. En outre, un État pourrait refuser d'accorder des privilèges et immunités aux diplomates, ce qui n'est guère concevable (Philippe, 1954).

\section{Relativité des privilèges et immunités diplomatiques}

Les développements sous cette rubrique examinent les limites des privilèges et immunités octroyés au corps diplomatique par la Convention de Vienne de 1961 que la pratique diplomatique a permis de révéler. C'est ainsi que devant les préoccupations souverainistes ou d'ordre public, les privilèges et immunités tombent.

\section{1 Prépondérance de la sécurité et de l'ordre public sur l'inviolabilité}

Le dictionnaire Larousse donne la définition de l'inviolabilité comme étant la protection spéciale accordée à certaines personnes (représentants diplomatiques) et à certains biens ( ambassade, archives diplomatiques et consulaires, etc.). Les avantages reconnus aux diplomates par le droit international ne les exonèrent pas de l'obligation de se conformer à la législation nationale de l'État d'accueil (art.41). Si l'inviolabilité est reconnue par le droit international, encore ne l'est-elle que dans certaines limites. À ce sujet, le courant doctrinal majoritaire soutient que l'inviolabilité ne peut empêcher l'État accréditaire d'adopter les mesures indispensables pour la sauvegarde de sa sécurité et de son ordre public si la conduite des agents diplomatiques est susceptible de les mettre en danger (Salmon, 1977). Au regard de l'importance de ces privilèges d'inviolabilité dans l'exécution des missions diplomatiques, le droit international pose des principes pour légaliser les limitations qui lui seraient apportées. À cet effet, l'inviolabilité peut être invoquée :

- En cas de légitime défense de la part des particuliers contre des actes commis par les personnes mêmes qui jouissent du privilège ;

- En cas de risques courus par l'une desdites personnes volontairement ou sans nécessité ;

- En cas d'actes répréhensibles, commis par elles, et provoquant de la part de l'État accréditaire des mesures de défense et de précaution. Hormis les cas d'urgence extrême, l'État accréditaire doit se borner à signaler les faits au gouvernement du diplomate, à demander sa punition ou son rappel et à faire, s'il y a lieu, cerner son hôtel pour empêcher des communications ou manifestations illicites (Annuel de l'institut du droit international, 1895). En somme, exception faite des 
cas d'extrême urgence, aucune contrainte directe ne peut être exercée contre la personne.

Les cas d'arrestation suivis d'expulsion sont fréquents dans la pratique contemporaine à la suite de prise en flagrant délit d'espionnage. Mais aussi, des arrestations temporaires ont été observées à propos de crimes et délits divers. Il va de soi que les voies de fait commis par les diplomates peuvent justifier leur arrestation temporaire, voire leur expulsion, le tout devant s'effectuer sans violences. Toutefois, on peut toujours craindre que l'arrestation ou l'expulsion de l'agent diplomatique soit purement injustifiée. Craignant des restrictions injustifiées qui aboutiraient à excès de cette nature, les auteurs de la Convention de Vienne ont délibérément omis de mentionner les limites de l'inviolabilité et l'ont plutôt présentée comme absolue (Salmon, 1977).

La pratique diplomatique confirme que le respect des règles du pays accréditant prime sur l'inviolabilité. Lorsque Julian Assange, de nationalité australienne et fondateur de Wikileaks, a fait l'objet d'inculpation de piratage informatique par le gouvernement américain et s'est réfugié à l'Ambassade de l'Équateur accréditée à Londres où il a vécu plus de 6 ans et a fini par se naturaliser équatorien, il bénéficiait de l'inviolabilité diplomatique dont les locaux de l'ambassade étaient couverts. Mais lorsque les autorités équatoriennes ont mis fin à son statut d'asile pour violations répétées aux règles régissant les conditions d'asile, la police britannique a pénétré dans l'ambassade d'Équateur à Londres, car ce pays ayant déjà mis fin au statut d'asile dont bénéficiait le fondateur de Wikileaks. Pour qu'il y ait une intervention des forces de l'ordre du pays d'accueil, il faut au préalable un consentement avec le chef de la mission (Convention de Vienne de 1961, art. 22.1). Ce cas illustratif confirme que les immunités dont jouit l'agent diplomatique ne lui garantissent pas l'impunité. Qui plus est, la communauté internationale a prévu la renonciation à ces immunités par l'État d'envoi. (Convention de Vienne de 1961, art. 32.1).

\section{III2. Étendue des privilèges et immunités en cas de possession de résidence permanente et/ou de nationalité du pays accréditaire}

Ces privilèges et immunités diplomatiques, qualifiés de ratione personae, connaissent des limites quant à la personne de l'agent diplomatique. Leur étendue peut être restreinte si l'agent diplomatique a la nationalité de l'État accréditaire ou possède sa résidence permanente sur le territoire de l'État accréditaire. Toutefois, la pratique n'est pas uniforme et l'opinion des auteurs diffère. Pour certains, un tel agent doit bénéficier de la plénitude des privilèges et immunité à moins que l'État accréditaire n'ait fait des réserves au moment de l'agrément. Pour d'autres, il ne doit bénéficier que des privilèges et 
immunités qui lui ont été expressément attribués par l'État accréditaire. Mais, la Commission de droit international et, à la suite, la Conférence de Vienne se sont prononcées en faveur d'une solution intermédiaire. Elles ont estimé qu'un minimum d'immunités était nécessaire pour remplir utilement la fonction et que le minimum devait être constitué par l'inviolabilité et l'immunité de juridiction pour les actes officiels accomplis dans l'exercice de ses fonctions (Salmon, 1977). Ce raisonnement emboite le pas à la Convention de Vienne qui stipule: " $\mathrm{A}$ moins que des privilèges et immunités supplémentaires n'aient été accordés par l'État accréditaire, l'agent diplomatique qui a la nationalité de l'État accréditaire ou y a sa résidence permanente ne bénéficie de l'immunité de juridiction et de l'inviolabilité que pour les actes officiels accomplis dans l'exercice de ses fonctions » (art. 38 alinéa 1).

Pour les membres du personnel de la mission diplomatique et les domestiques privés ayant la nationalité de l'État accréditaire ou possédant leur résidence permanente sur le territoire de cet État, ils ne bénéficient de privilèges et immunités que dans la mesure où l'État accréditaire leur en reconnaît. (Salmon, 1977).

Se référant toujours à la Convention de Vienne, les membres de la famille de l'agent diplomatique n'auront de privilèges et immunités que dans la mesure où l'Etat accréditaire les leur reconnaitra (art. 38 alinéa 2). Mais il s'agit bien là d'un minimum et il est toujours loisible à l'État accréditaire d'offrir plus.

\section{3. La temporalité des privilèges et immunités diplomatiques}

Les privilèges et immunités diplomatiques sont aussi marqués par le caractère ratione temporis, qui explique leurs limites dans le temps. La question est alors de savoir à partir de quel moment les privilèges et immunités peuvent-ils être revendiqués et à quel moment cessent-ils de porter leurs effets ? Relativement au point de départ de ces privilèges et immunités, l'État accréditaire en est débiteur aussitôt que le bénéficiaire apparaît sur son territoire en sa qualité officielle. Ce sera donc au moment où il pénètre sur le territoire de l'État accréditaire pour regagner son poste qu'il sera revêtu de sa qualité officielle. C'est la connaissance, par les autorités locales, de la qualité de diplomate qui compte et non la formalité de remise des lettres de créance. L'arrivée étant toujours notifiée auparavant, si le diplomate arrive de l'étranger, il aura été annoncé ou sera porteur de visas diplomatiques du pays accréditaire dont un représentant du Ministère des Affaires étrangères l'attendra éventuellement à la gare ou à l'aérodrome ou au port d'arrivée. Il n'est, bien entendu, pas nécessaire d'attendre pour cela la remise des lettres de créance. Pour jouir des privilèges et immunités diplomatiques, l'audience solennelle de remise des lettres de créance n'est pas une formalité essentielle. Elle peut avoir lieu après plusieurs semaines. Il en est ainsi, car, si la remise 
des lettres de créance était une exigence fondamentale, seuls les chefs de mission qui remettent, les lettres jouiraient des privilèges et immunités ; ce qui n'est pas le cas (Salmon, 1977).

$\mathrm{Au}$ cas où le diplomate se trouve déjà sur le territoire de l'État accréditaire, la Convention de Vienne de 1961 prévoit que les privilèges et immunités lui sont accordés au moment où la nomination aura été officiellement notifiée au Ministre des Affaires étrangères (Salmon, 1977; Art. 39 de la convention de Vienne). S'agissant du moment où les privilèges et immunités des agents diplomatiques cessent de produire leurs effets, c'est en principe à l'expiration de la mission. Mais, les agents en fin de mission continuent d'en bénéficier pour une période de temps raisonnable nécessaire à l'agent pour liquider ses affaires et quitter le territoire de l'État accréditaire. Par ailleurs, les privilèges et immunités subsistent en ce qui concerne les actes accomplis dans l'exercice de ses fonctions comme membre de la mission diplomatique. (Salmon, 1977).

\section{4. Circonscription spatiale des privilèges et immunités diplomatiques}

Les privilèges et immunités diplomatiques dans leur dimension ratione loci connaissent des limites dans l'espace. Sur le territoire de l'État accréditant, l'agent diplomatique est un citoyen ordinaire comme les autres. Par conséquent, ces privilèges et immunités ne produisent aucun effet. Il peut même y être assigné. Sur le territoire de l'État tiers, les privilèges et immunités produisent des effets à condition que le passage de l'agent diplomatique trouve son fondement dans l'accomplissement de fonctions officielles. On parle souvent de l'agent diplomatique en transit. (Salmon, 1977). L'Institut de droit international a pris position en ce sens en affirmant que les privilèges et immunités s'exercent, tant à l'aller qu'au retour dans les pays que l'agent doit traverser, soit pour gagner son poste ou quitter son poste, soit pour rentrer temporairement dans son pays d'origine. (Art. 5 de la Résolution de l'Institut de droit international sur les immunités diplomatiques, New York, 1929). La même solution est admise de façon très large par la Convention de Vienne.

Les États tiers ne doivent pas entraver le passage sur leur territoire des membres du personnel administratif, technique et de service de la mission. Il en est de même pour les membres de leur famille notamment ceux bénéficiant des privilèges et immunités lorsqu'ils accompagnent l'agent diplomatique ou lorsqu'ils voyagent séparément pour le rejoindre ou pour rentrer dans leur pays.

\section{5. Les contrôles sur la personne et les fouilles de bagages de diplomates dans les aéroports}

Les fouilles des diplomates ainsi que le contrôle de leurs bagages dans les aéroports ont soulevé au cours des dernières décennies plusieurs différends 
entre les États concernés. La question de fond porte en ce domaine sur l'interprétation des dispositions de la convention de Vienne sur les relations internationales du 18 avril 1961 qui ont trait à la question de la fouille des bagages personnels de l'ambassadeur et des membres de sa famille.

La difficulté naît de ce que les États admettent désormais que la sécurité des aéroports et des passagers des avions de ligne passe par un «modus vivendi » entraînant une certaine relativisation des dispositions de la convention de Vienne et qu'il puisse y avoir un contrôle des bagages des personnes concernées, voir même une fouille au corps.

Pourtant, les dispositions de la Convention de Vienne sur les relations diplomatiques prévoient que l'agent diplomatique ainsi que les membres de sa famille sont exemptés de l'inspection de leurs bagages personnels, «à moins qu'il n'existe des motifs sérieux de croire » qu'ils contiennent des objets autres que ceux destinés à l'usage officiel de la mission ou à l'usage personnel de l'agent et des membres de sa famille, ainsi que des objets dont l'importation ou l'exportation est interdite par la législation de l'État accréditaire ou soumise à ses règlements de quarantaine. (art $36 \mathrm{al} .2$ et $37 \mathrm{al} .1$ ).

Les agents diplomatiques et les membres de leur famille peuvent donc, en principe, exiger que leurs bagages ne soient pas inspectés. Toutefois, prévention contre les attaques terroristes oblige, la pratique peut s'avérer tout autre selon les Etats, mais aussi le degré de réaction autant que le manque de discernement manifestés par les agents de sécurité des aéroports dans l'application des instructions qui leur sont données. Elle est donc devenue assez diversifiée dans ce domaine. Dans les aéroports de certains États, la fouille des bagages personnels des agents diplomatiques et des membres de leur famille aux passagers des portiques de sécurité est systématique et entraîne régulièrement des plaintes auprès des services du Protocol local. À titre d'exemple, en mars 2014, M. Salaheddine Mezouar, ministre des Affaires étrangères du Maroc, lors du transit à Roissy - Charles - de - Gaulle, a dû, comme d'autres passagers, enlever veste, ceinture, chaussettes et chaussures, ce qui a contraint ensuite le ministre français des Affaires étrangères M. Laurent Fabius, à présenter au Maroc les excuses officielles de la France (Annuaire français de relations internationales, 2015, volume XVI). Cela étant, il est bien difficile dans le contexte international actuel de s'y opposer. Et ce sont également les compagnies aériennes qui peuvent demander à ce que tous les passagers soient fouillés : la personne elle-même et ses bagages. Ils restent aux agents de la sécurité à agir avec discernement.

\section{6. Protection incidente des correspondances et valises diplomatiques}

La valise diplomatique désigne un moyen de transport utilisé pour échanger différents objets sous couvert de l'immunité diplomatique (Bezut, 2018). Aux termes de la Convention de Vienne, les valises diplomatiques ou 
consulaires doivent permettre aux gouvernements de correspondre avec les missions et, à celles-ci, de correspondre entre elles par des envois scellés. Les valises qui ne peuvent contenir que la correspondance, des documents ou objets de caractère officiel, doivent porter des marques extérieures visibles permettant leur identification, être revêtues du sceau de l'État d'envoi (ou de la mission), lequel doit en assurer une fermeture inviolable. La valise ou la correspondance ne peut être ni ouverte, ni retenue, ni soumise à des contrôles électroniques ou par rayons X. Toutefois, «[si] les autorités compétentes de l'État de résidence ont de sérieux motifs de croire que la valise consulaire contient d'autres objets que la correspondance, les documents et les objets visés [par la convention de Vienne de 1963], elles peuvent demander que la valise soit ouverte en leur présence par un représentant autorisé de l'État d'envoi » (Convention de Vienne de 1963, art. 35,3).

Les États tiers accordent à la correspondance et aux autres communications officielles en transit, y compris les messages en code ou en chiffre, la même liberté et protection que l'État accréditaire.

Ils accordent aux courriers diplomatiques, auxquels un visa de passeport a été accordé si ce visa était requis, et aux valises diplomatiques en transit la même inviolabilité et la même protection que l'État accréditaire est tenu de leur accorder. Les mêmes obligations restent inchangées lorsque leur présence sur le territoire de l'État tiers est due à la force majeure (Convention de Vienne de 1961, art. 40,1).

\section{Particularisme de la protection des locaux des missions diplomatiques et de l'imposition}

La logique qui guide l'octroi des privilèges et immunités au corps diplomatique, à savoir offrir les conditions nécessaires aux agents diplomatiques pour leur permettre d'accomplir efficacement leurs fonctions en tant que représentants des États, constitue le fondement de l'inviolabilité des locaux de la mission diplomatique. Il n'est pas permis aux agents de l'État accréditaire d'y pénétrer, sauf avec le consentement du chef de la mission même si la Convention de Vienne de 1961 sur les relations diplomatiques reste muette sur la façon dont le consentement doit être donné.

\section{Conclusion}

$\mathrm{Au} \mathrm{vu}$ des analyses précédentes, les privilèges et immunités diplomatiques constituent des outils mis à la disposition des agents diplomatiques pour leur permettre d'exécuter des fonctions à eux confiées par les gouvernements accréditant. Le diplomate n'en tire avantage qu'en sa qualité de représentant officiel de l'État accréditant. C'est ce but d'intérêt général qui guide l'octroi de ces privilèges et immunités. Puisque le but est d'accomplir en toute quiétude les missions de la République, la pratique 
étatique et même conventionnelle garantit ces privilèges et immunités aux membres de la famille de l'agent diplomatique vivant ou se déplaçant avec lui. Ils permettent aux diplomates de mieux servir et protéger les intérêts de l'État qu'ils représentent sans se soucier de leurs intérêts personnels. Étant donné qu'aussitôt relevés de leurs fonctions, ils en perdent le bénéfice, il s'en suit qu'ils sont détachés de la personne de l'agent diplomatique et que leurs motivations fondamentales et de faciliter l'accomplissement de leurs missions diplomatiques ou du moins leurs fonctions et non pour leur conférer des avantages personnels.

La prédominance de l'attachement des privilèges et immunité à la fonction ou mission du diplomate n'exonère pas ce dernier des comportements illégaux dont il pourrait être coupable. C'est la raison de l'existence de leurs limites. En cas d'abus de ces privilèges et immunités diplomatiques, l'agent qui s'en est rendu coupable risque d'être déclaré persona non grata, d'expulsion ou de rappel.

Ainsi, les privilèges et immunités reconnus au corps diplomatique sont loin d'être absolus. Des limites s'observent sur différentes dimensions. Il y a des limites admises coutumièrement, des limites liées à la personne de l'agent diplomatique, des limites dans le temps et dans l'espace. De telles limites permettent d'éviter les abus que peuvent commettre les agents diplomatiques volontairement ou involontairement quoique présentant parfois des risques d'entraver la mission ou les fonctions des agents diplomatiques.

Les États fonctionnent en concurrence, c'est la raison pour laquelle les privilèges et immunités diplomatiques sont octroyés selon le principe de la réciprocité. Chaque État les accorde dans un but de faciliter les fonctions des missions diplomatiques et dans le désir de voir bénéficier une situation identique. Cela permet à chaque État de se sentir protégé dans ses intérêts lorsqu'il s'engage dans les relations diplomatiques.

Les états accordent ces privilèges et immunités diplomatiques sur base des dispositions de la Convention de Vienne sur les relations diplomatiques de 1961. Ce texte a subi l'usure du temps et certaines de ses dispositions ne correspondent plus à la réalité actuelle. Cela est le cas notamment de l'interdiction d'installer et d'utiliser un poste émetteur de radio qu'avec l'assentiment de l'État accréditaire (Convention de Vienne de 1961 art. 27.1). Cette interdiction trouvait son fondement dans le fait que l'existence des techniques d'installation et de transmission a posé à l'époque le problème de coût. Les petits États ne se sont pas montrés enthousiastes, l'égalité réelle des moyens étant en cause, car seules les grandes puissances sont en mesure de procéder à une telle installation. Mais cela n'est pas le cas aujourd'hui. Également, la liberté de passage que l'État tiers accorde à l'agent diplomatique en transit (art. 40) importe peu. Aujourd'hui ces agents se déplacent en avion lorsqu'ils veulent quitter un territoire vers un autre. 
Par ailleurs, en mettant en application la règle de la réciprocité, les États risquent d'acquérir une tendance à décider unilatéralement quels sont les privilèges qu'ils accordent aux diplomates étrangers et quels sont ceux qu'ils leur refusent. Une telle attitude serait contraire au droit diplomatique. Ce risque tient au fait que les règles relatives aux privilèges sont des normes réciproques en ce qui concerne les privilèges de courtoisie, c'est-à-dire les privilèges qui ne sont pas nécessaires à l'accomplissement des fonctions du diplomate. Par contre pour les privilèges qui sont essentiels à cet accomplissement, comme pour l'inviolabilité, les États devraient accorder ces privilèges et immunités indépendamment de la manière dont un autre État les accorde. Si ce dernier agit de manière illicite, l'État pourra recourir aux sanctions prévues par le droit international et notamment à la rupture des relations diplomatiques. Le respect mutuel des engagements envers les diplomates permet aux pays d'éviter les incidents diplomatiques.

\section{References:}

1. Bély L. (2012). Pour l'histoire des relations internationales. Presse Universitaire de France.

2. Burlamaqui J.J. (1766). Principe du droit de la nature et des gens, T.VIII, Genève.

3. Bezut M. (2018), Valise diplomatique, dans Hugues Moutouh (dir.) et Jérôme Poirot (dir.), Dictionnaire du renseignement, Paris.

4. 4.Casseses A. et Delmas-Marly S. (2002). Crimes internationaux et juridictions nationales. Ed. Presses universitaires de France, Paris.

5. De landesheere G. (1975). Introduction à la recherché en éducation, 5e éd. Entreprise Moderne, ESF. Paris.

6. Duffar J. (1982).Contribution à l'étude des privilèges et immunités des organisations internationales, L.G.D.J, Paris.

7. Fauchille P. (1922). Traité de droit international, Tome 1 première partie, Librairie Arthur Rousseau, Paris.

8. Fedele D. (2017), Naissance de la diplomatie moderne (XIIIe-XVIIe siècles). L'ambassadeur au croisement du droit, de l'éthique et de la politique, Baden-Baden - Zürich/St. Gallen, Nomos - Dike Verlag

9. Festinger L.et Katz D. (1977). Les méthodes de recherche en sciences sociales, T.2, PUF. Paris.

10. Gentilis, A. (1585). De Legationi bus libritres.

11. Guggenheim P. (1953). Traité de droit international public, vol. I, Genève.

12. Guillaume G. (2001). Dictionnaire de droit international public, Bruylant, Bruxelles.

13. Grotius H. (1724). Le droit de la guerre et de la paix, L.II, Amsterdam.

14. Hurst C. (1926). Les immunités diplomatiques, L.II, RCADI. 
15. Keith H. \& Richard L. (1995). The Practice of Diplomacy: Its Evolution, Theory and Administration, Routledge, London.

16. Kissinger H. (trad. de l'anglais par Marie-France de Paloméra), (1996), Diplomatie, Fayard, Paris.

17. Léon A. (1977). Manuel de psychologie expérimentale, PUF. Paris.

18. Loubertdel bayle J. L. (1978). Introduction aux méthodes des sciences sociales, Toulouse, Privat.

19. Mucchielli R. (1977). Analyse de contenu des documents et des communications, Editions ESF, Paris.

20. Nguyen quocdinh, Daillier P. et Pellet A. (1999). Droit international public, Formation du droit, sujets, relations diplomatiques et consulaires, responsabilité, règlement des différends, maintien de la paix, espaces internationaux, relations économiques, environnement, 6e éd. L.G.D.J, Paris.

21. Papini R. et Cortese G. (1972). La rupture des relations diplomatiques et ses conséquences, Pedone, Paris.

22. Pradier-Fodere P. (1899). Cours de droit diplomatique, vol.1, Paris.

23. Philippe C. (1964). Le droit diplomatique contemporain, 2e éd., Genève.

24. Gentilis, A. (1585). De Legationi bus libritres.

25. Roger P. et Grawitz M. (1964). Méthodes des sciences sociales, T.2, paris, Dalloz, Paris.

26. Rongere P. (1977). Méthodes des sciences sociales, Dalloz, 3e éd., Paris.

27. Salmon J.A. (1977).Fonctions diplomatiques, consulaires et internationales, T.I, 3e éd., Presses universitaires de Bruxelles, Bruxelles.

28. Salmon J.A. (1976-1977).Fonctions diplomatiques, consulaires et internationales, T.II, 3e éd. Presses universitaires de Bruxelles, Bruxelles.

29. Vattel E. (1830). Le droit des gens, T.13, L.IV, Paris.

\section{Textes juridiques régionaux et internationaux}

30. Privileges and Immunities for the Diplomatic Corps and International and Regional Organization in Uganda, March, 2012.

31. Convention de Vienne sur les relations diplomatiques du 18 avril 1961.

32. Résolution de l'Institut de droit international sur les immunités diplomatiques, New York, 1929.

\section{Autres documents}

33. Annuaire de la Commission du droit international, vol. II, NationsUnies, 28 avril 4 juillet 1958. 
34. Annuaire de l'Institut de droit international, Vol.14, Cambridge, août 1895.

35. Annuaire français de relations internationales, volume XVI, Université Panthéon-Assas, Centre Thucydide, 2015.

36. Pr. NZOHABONAYO A. Notes de cours, Séminaire des Mémorants, École Nationale d'Administration, Bujumbura, 2019.

\section{Webographie}

37. https://legal.un.org (page visitée le 13 novembre 2020 à 11 h 30).

38. https://fr.wikipedia.org/wiki/Droit_de_1\%C3\%A9gation\#cite_note-1 (page visitée le 13 novembre 2020 à 11 h 30).

39. https://fr.wikipedia.org/wiki/Julian_Assange (page visitée le 2 septembre 2020 à 22 h 30).

40. https://www.jeuneafrique.com/dossiers/affaire-khashoggi-londe-dechoc/ (page visitée le 30 octobre 2020 à 22 h 30).

41. https://www.linternaute.fr/dictionnaire/fr/definition/inviolabilite/ (page visitée le 17 septembre 2020 à 7 h 50). 